Article

\title{
Energy Management Strategy of a Novel Electric Dual-Motor Transmission for Heavy Commercial Vehicles Based on APSO Algorithm
}

\author{
Jiajia Liang ${ }^{1,2,3}$, Xiangyang Xu ${ }^{1,2}$, Peng Dong ${ }^{1,2}$, Tao Feng ${ }^{3}$, Wei Guo ${ }^{2, *}$ and Shuhan Wang ${ }^{1,2, *}$ \\ 1 School of Transportation and Science Engineering, Beihang University, Beijing 102206, China; \\ jiajialiang@buaa.edu.cn (J.L.); xxy@buaa.edu.cn (X.X.); peng.dong@buaa.edu.cn (P.D.) \\ 2 Ningbo Institute of Technology, Beihang University, Ningbo 315832, China \\ 3 Transmission Department, eKontrol Co., Ltd., Suzhou 215211, China; fengtao106@126.com \\ * Correspondence: guowqd@126.com (W.G.); wsh@buaa.edu.cn (S.W.)
}

Citation: Liang, J.; Xu, X.; Dong, P.; Feng, T.; Guo, W.; Wang, S. Energy Management Strategy of a Novel Electric Dual-Motor Transmission for Heavy Commercial Vehicles Based on APSO Algorithm. Sustainability 2022, 14, 1163. https://doi.org/ $10.3390 /$ su14031163

Academic Editor: Eckard Helmers

Received: 23 December 2021

Accepted: 18 January 2022

Published: 20 January 2022

Publisher's Note: MDPI stays neutral with regard to jurisdictional claims in published maps and institutional affiliations.

Copyright: () 2022 by the authors. Licensee MDPI, Basel, Switzerland. This article is an open access article distributed under the terms and conditions of the Creative Commons Attribution (CC BY) license (https:// creativecommons.org/licenses/by/ $4.0 /)$.

\begin{abstract}
With the development of electric vehicles, dual-motor transmission has become a potential alternative for automated manual transmission (AMT) due to the solution of power interruption and the improvement of energy efficiency. In this paper, a novel electric dual-motor transmission (eDMTP) for heavy commercial vehicles is proposed. Then, a 4-layer energy management strategy is developed to optimize dynamics performance and energy efficiency. Subsequently, a real vehicle operation is performed to validate the control strategy and the performance of eDMTP. The results demonstrate that the operating points of the two motors are both in and around the high-efficiency area under normal mode. This research lays the foundation for the development of a pure electric vehicle transmission system.
\end{abstract}

Keywords: dual-motor; transmission; mode switching; energy management strategy; torque split; control strategy

\section{Introduction}

The issue of commercial vehicle emission has been the focus of attention in the transportation field because of the high proportion of carbon dioxide and nitrogen oxides. To ameliorate environmental quality, many countries have put forward stricter emission regulations for commercial vehicles, especially for medium and heavy-duty vehicles [1]. Due to the advantages of zero emissions and low electricity costs, the pure electric vehicle is suitable for relatively fixed road conditions in specific commercial operating environments, such as mining trucks, port shuttles, and buses [2,3].

Relying on the maturity of traditional automated manual transmission (AMT) technology, 'single drive motor + multi-gear AMT' is adopted in pure electric commercial vehicles, which has a simple structure, high transmission efficiency, and can meet the application requirements of complex road conditions (freeway and off-road). However, interruption of power occurs during gear shifting. In order to enhance the shifting quality, control methods have been intensively studied by many researchers [4-7], but the shift torque interruption cannot be completely eliminated via optimizing control strategies. For the purpose of ensuring vehicle safety and reliable operation, shifting is not allowed under certain road conditions (such as heavy load uphill and downhill), which reduces the amount of energy feedback and is not conducive to the energy saving of the vehicle.

Dual-motor transmissions have been explored by many researchers in recent years to deal with the challenge of power interruption during gear shifting and effectively improve vehicle energy utilization [8-10]. However, the focus is mainly on passenger car applications. There are still few studies on heavy commercial electric vehicles. Two 
typical dual-motor configurations have been studied by some investigators. The dualmotor coupling-propulsion and centralized drive systems proposed by some authors could reduce the mode switching shock and improve the dynamic performance of electric vehicles [11-13]. Zhang et al. [11] used electromechanical coupling control to realize the dynamic coupling of the distributed drive system, which not only enhanced the high-speed stability of the vehicle, but also coordinated the torque output of the drive system, thereby suppressing the driving torque fluctuation and reducing the work intensity of the motor and the controller. They also proposed a staged multivariable combined control method and reduced the shock generated by the mode switching to below $10 \mathrm{~m} / \mathrm{s}^{3}$ [12]. Park et al. [13] developed a control algorithm of dual-motor torque vectoring system. By some case simulations, it was verified that the handling performance of vehicles was improved in agile and safe modes. However, the system significantly increases the complexity and cost of the vehicle. The other configuration is the combination of two electric motors by a planetary gear train. The advantage of this structure is that it is more efficient than a single motor [14-16]. Under the same total power, maximum speed, and maximum torque, the average efficiency of two motors combined via planetary gear train in the power supply process is $90.13 \%$, while the efficiency of a single motor is $82.43 \%$ [14]. Mantriota et al. [15] investigated the feature of single-planet and double-planet sets and the results show that the power transmission performance of the dual motors is generally better than that of the single motor. The average efficiencies of both the regeneration phases and power delivery are improved by about $9 \%$. However, the coupled dual-motor operating points lead to suboptimal energy-saving effects. Thus, it is necessary to explore a new transmission configuration that can eliminate power interruption and completely decouple the working status of the dual motors, thereby reducing product power consumption as much as possible.

Energy management strategies for multi-power transmissions have attracted considerable attention, especially for hybrid transmissions [17-21]. These strategies aim to determine the operational matters of each power source and reasonably distribute the torque, thereby improving the efficiency of the system and reducing the energy consumption of vehicles. At present, a rule-based energy management strategy is widely used by designing logical thresholds (the battery state of charge, vehicle demand torque, etc.) [22-24], but this method relies heavily on practical experience and is not economical. The global optimization algorithm can receive the lowest theoretical energy consumption value, and the operating road conditions are required as input conditions [25-27]. Wang et al. [25] demonstrated that the dynamic programming (DP) algorithm method could supply a $7-9 \%$ energy saving relative to that of a rule-based strategy. A DP-based optimal control algorithm was constructed by Wang et al. [26] for a plug-in hybrid electric vehicle. The results showed that the fuel saving ability was improved under the control algorithm. Due to the massive amount of calculation, the limited computing power of the existing controllers, and inconsistency with the actual operating conditions, it is usually used as a benchmark for other algorithms and rarely used in practice.

To address the problem of power interruption and further improve the energy-saving effect of vehicles, a new electric dual-motor transmission (eDMTP) is proposed in this work for heavy commercial vehicles. Based on eDMTP, a 4-layer energy management strategy is designed, and each layer is described in detail. A real-time torque split control strategy is developed based on the adaptive particle swarm algorithm (APSO), and a dual-motor power scale factor is introduced to reduce the amount of calculation. Finally, the performance of eDMTP and energy management strategy are verified and analyzed by actual vehicle operation.

This work is organized as follows. Section 2 introduces the transmission scheme of eDMTP system. In Section 3, the four-layer energy management strategy and the optimal torque split strategy are designed. In Section 4, the performance of the transmission and control strategy are verified by real-vehicle tests. Finally, conclusions are summarized in Section 5 . 


\section{2. eDMTP System}

The schematic diagram of eDMTP is illustrated in Figure 1 and the concept of symmetrical design is adopted. The power of the two high-speed motors (EM1 and EM2) is transmitted to the output shaft through two intermediate shafts. The operating points of the dual motors are wholly decoupled, which allows both motors to work in a high efficiency area and is conducive to improving the economy of transmission. The power take-off (PTO) and mechanical oil pump (MOP) units, connected through intermediate shaft, are driven by EM1 and the two integrated mode switching actuators are respectively controlled by two small direct current motors to realize the movement and shifting of the four dog clutches (A, B, C and D). In order to meet the power requirements of larger tonnage vehicles (full load of 150 tons), a low-speed drive motor can be selectively matched on the output shaft of the transmission.

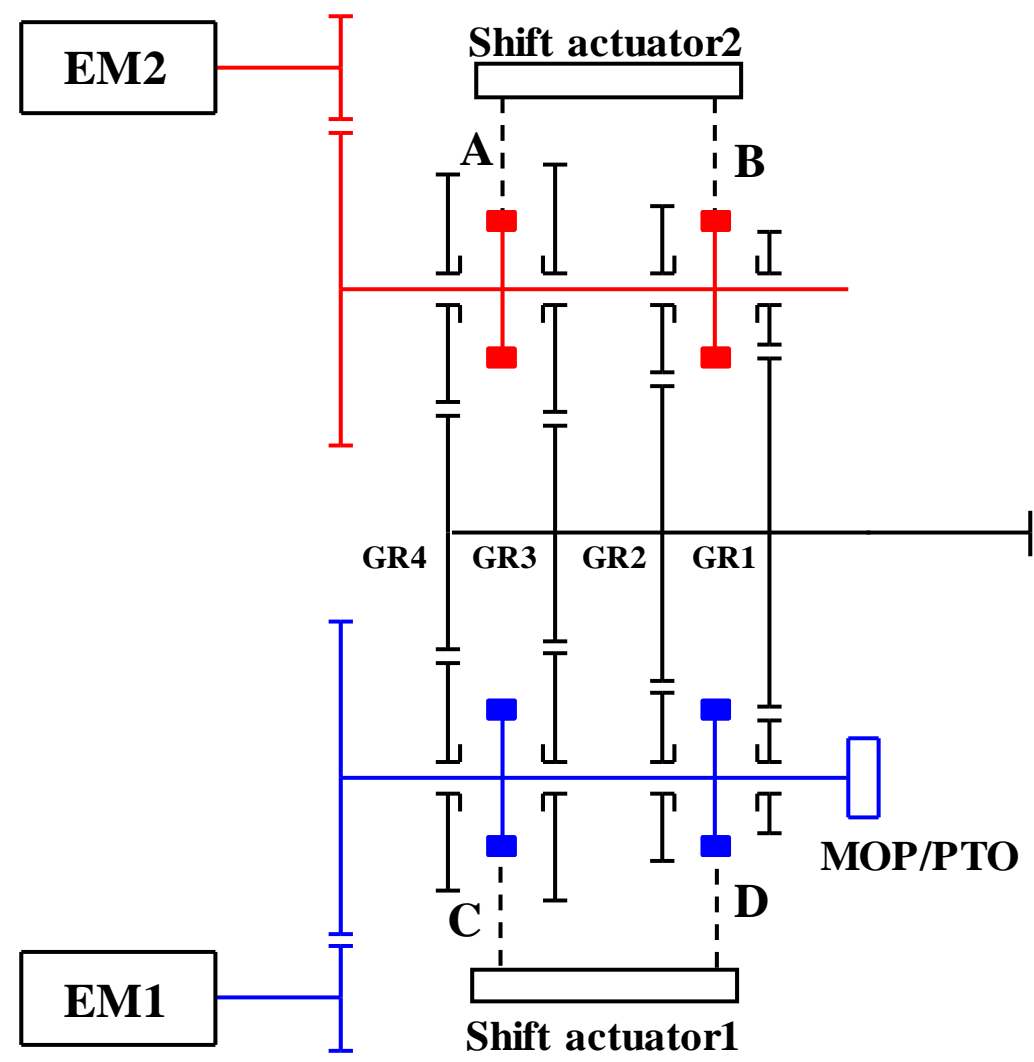

Figure 1. The transmission scheme of eDMTP.

The operating mode and switching logic of eDMTP are shown in Figure 2. It contains a total of 7 dual-motor modes (M1-M7). Among them, M1 and M2 are the escape modes, which can meet the greater torque demand of the vehicles under specific road conditions (such as muddy and larger slope roads), and M3-M7 are normal modes for regular driving. The gear ratio is decreased from M1 to M7. Therefore, the maximum output torque and the maximum speed can be obtained in M1 and M7, respectively. A single motor mode, between every two dual motor modes, can ensure that a motor always provides the driving force to the vehicle during the mode switching process, thereby eliminating power interruption. 


\begin{tabular}{|c|c|c|c|c|c|c|c|c|c|c|c|c|c|c|c|}
\hline \multirow{2}{*}{\multicolumn{2}{|c|}{ Modes }} & \multicolumn{3}{|c|}{ Clutch A } & \multicolumn{3}{|c|}{ Clutch B } & \multicolumn{3}{|c|}{ Clutch C } & \multicolumn{3}{|c|}{ Clutch D } & \multicolumn{2}{|c|}{ Gear Ratio } \\
\hline & & $\mathbf{L}$ & $\mathbf{M}$ & $\mathbf{R}$ & $\mathbf{L}$ & $\mathbf{M}$ & $\mathbf{R}$ & $\mathbf{L}$ & $\mathbf{M}$ & $\mathbf{R}$ & $\mathbf{L}$ & $\mathbf{M}$ & $\mathbf{R}$ & EM2 & EM1 \\
\hline \multirow[t]{2}{*}{ M0 } & $0-0$ & & 0 & & & - & & & 0 & & & - & & I & I \\
\hline & 1-0 & & - & & & & - & & 0 & & & 0 & & 12.1 & I \\
\hline \multirow[t]{2}{*}{ M1 } & $1-1$ & & - & & & & - & & $\bullet$ & & & & 0 & 12.1 & 14.3 \\
\hline & $0-1$ & & - & & & • & & & 0 & & & & 0 & I & 14.3 \\
\hline \multirow[t]{2}{*}{ M2 } & $2-1$ & & - & & ○ & & & & - & & & & ○ & 7.1 & 14.3 \\
\hline & $2-0$ & & - & & - & & & & • & & & $\bullet$ & & 7.1 & I \\
\hline \multirow[t]{2}{*}{ M3 } & $2-2$ & & - & & - & & & & 0 & & - & & & 7.1 & 8.4 \\
\hline & $0-2$ & & - & & & ○ & & & 0 & & - & & & I & 8.4 \\
\hline \multirow[t]{2}{*}{ M4 } & $3-2$ & & & $\bullet$ & & • & & & $\bullet$ & & - & & & 4.7 & 8.4 \\
\hline & 3-0 & & & - & & - & & & - & & & - & & 4.7 & I \\
\hline \multirow[t]{2}{*}{ M5 } & 3-3 & & & - & & ○ & & & & 0 & & $\bullet$ & & 4.7 & 5.6 \\
\hline & $0-3$ & & - & & & • & & & & - & & $\bullet$ & & I & 5.6 \\
\hline \multirow[t]{2}{*}{ M6 } & $4-3$ & 0 & & & & • & & & & - & & $\bullet$ & & 2.0 & 5.6 \\
\hline & 4-0 & - & & & & • & & & 0 & & & $\bullet$ & & 2.0 & I \\
\hline M7 & 4-4 & 0 & & & & - & & - & & & & $\bullet$ & & 2.0 & 3.1 \\
\hline
\end{tabular}

L: Left position; M: Middle position; R: Right position; ๑ : Engaged

Figure 2. The operating modes and working logic of eDMTP.

From the perspective of permutation and combination, the dual-motor mode realized by two separately controlled shifting actuators is much higher than the defined seven types. In some modes, the operating points of EM1 and EM2 are significantly different, resulting in a serious imbalance of the force acting on the output shaft and a decrease in system stability. Therefore, the two shifting actuators alternately perform mode up/down actions to realize the steady operation of transmission.

\section{Energy Management Strategy}

In the power split of dual-motor transmissions, energy management strategies are critical to enhance dynamic performance and optimize energy efficiency. From the perspective of system control, the energy management strategy of eDMTP consists of four layers, namely driver torque demand, operating mode decision, optimal torque split, and torque arbitration. Figure 3 shows the overall architecture.

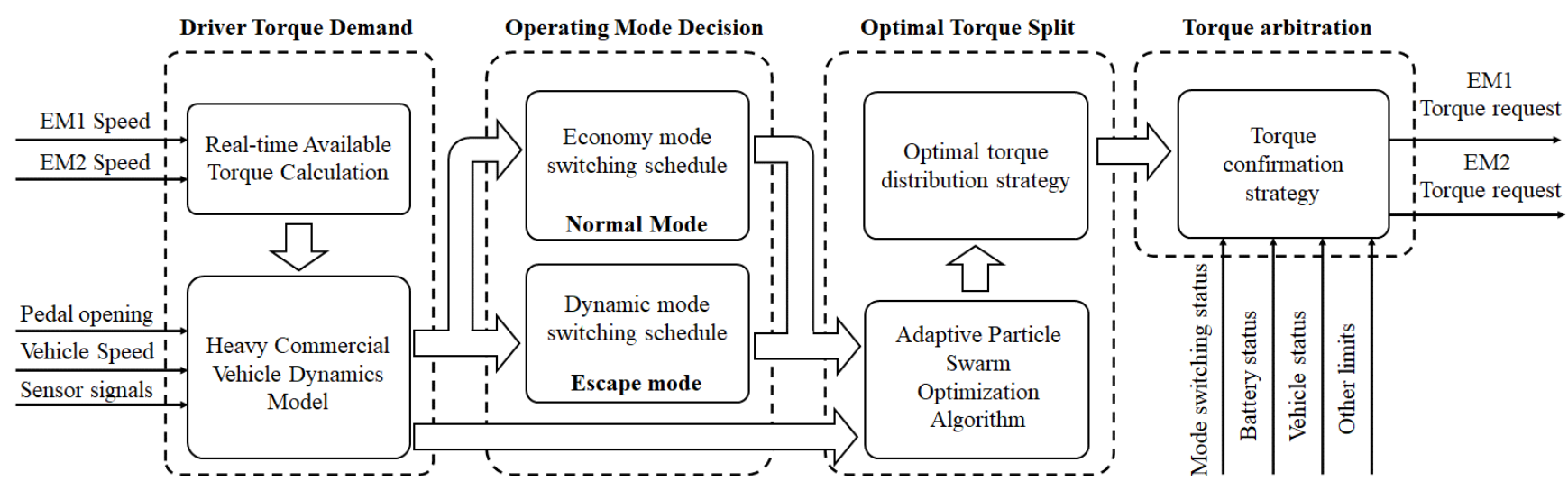

Figure 3. Energy management strategy flowchart. 


\subsection{Driver Torque Demand}

In order to determine the optimal operating mode of the transmission and torque of the dual motors in different modes, real-time calculation of the required torque of the vehicle under the driver input is the primary task. Based on the driving command (acceleration/brake pedal signal), the status of dual motors and other sensor signals (such as anti-lock brake system and displacement transducer), the layer calculates the required torque through the vehicle dynamics model. At the same time, according to the difference between the expected and the actual speeds, the Proportion Integration (PI) controller is used to calculate the compensation torque. Considering that the vertical and yaw motion of the vehicle has a smaller impact on the energy consumption of the vehicle, only the longitudinal dynamic model is established and represented by:

$$
T_{\text {out_req }}=\frac{\left(m_{\mathrm{V}} g \sin \theta+\frac{1}{2} \rho A_{\mathrm{V}} C_{\mathrm{d}} v^{2}+m_{\mathrm{V}} g C_{\mathrm{t}} \cos \theta\right) R_{\mathrm{W}}}{i_{f} \eta_{f}}
$$

where $m_{\mathrm{V}}$ and $A_{\mathrm{V}}$ are the vehicle mass and frontal area, respectively, and the units are $\mathrm{kg}$ and $\mathrm{m}^{2} ; C_{\mathrm{d}}$ and $C_{\mathrm{t}}$ denote the drag and tire rolling friction coefficients, respectively; $g$ denotes gravity acceleration, $\mathrm{m} / \mathrm{s}^{2} ; R_{\mathrm{W}}$ is the tire dynamic radius, $\mathrm{m} ; \theta$ is the slope angle of the road, degree; $v$ is the vehicle speed, $\mathrm{km} / \mathrm{h} ; \rho$ is the air density, $\mathrm{kg} / \mathrm{m}^{3} ; i_{f}$ is the final drive ratio; $\eta_{f}$ is the final drive efficiency; and $T_{\text {out_req }}$ is the output request torque of transmission, $\mathrm{Nm}$.

\subsection{Operating Mode Decision}

Determining the appropriate transmission mode in real time is a top priority for the decision layer. Based on the present operating state and the torque demand of the vehicle, the layer outputs the target mode and sends it to the mode management layer to perform the switching action by utilizing a robust control strategy. This strategy avoids the computation burden of transmission control unit (TCU) caused by the simultaneous optimization of the operation modes and the dual-motor operating points. Considering the particularity of mining vehicles, the layer first determines whether the vehicle needs to work in the escape mode (M1/M2). Because the vehicle must resolve the predicament in a short time, the escape mode follows the dynamic mode switching law. The operating mode is obtained through the economy mode switching law when the vehicle is driven in the normal mode. The rules for switching between escape mode and economy mode are obtained through offline calculations.

\subsection{Optimal Torque Split}

The torque of the dual motors can be calculated with appropriate operating mode obtained by Section 3.2. The optimal torque split strategy of dual motors includes optimal drive and braking torque distribution strategies. Different from the driving torque distribution strategy, the electric braking is given priority to maximize the energy feedback and reduce the number of recharges when the vehicle is braking. Furthermore, the mechanical braking is triggered only when the maximum electric braking cannot meet the vehicle's braking requirements. The torque split strategy of this layer is formulated without triggering mechanical braking.

\subsubsection{Optimization Problem Description}

\section{Determine Optimization Variable}

To reduce the amount of computation, the dual-motor power scale factor $(k)$ is introduced and considered as the optimized variable, defined as follows:

$$
k(s)=\frac{P_{\mathrm{EM} 1}(s)}{P_{\mathrm{EM} 2}(s)}=\frac{P_{\mathrm{EM} 1}(s)}{P_{\mathrm{des}}(s)-P_{\mathrm{EM} 1}(s)}
$$




$$
\begin{aligned}
& P_{\mathrm{EM} 1}(s)=T_{\mathrm{EM} 1}(s) n_{\mathrm{EM} 1}(s) \\
& P_{\mathrm{EM} 2}(s)=T_{\mathrm{EM} 2}(s) n_{\mathrm{EM} 2}(s)
\end{aligned}
$$

where $P_{\text {des }}(s)$ denotes the demand power of vehicle, KW; $P_{\mathrm{EM} 1}(s)$ and $P_{\mathrm{EM} 2}(s)$ denote the power of EM1 and EM2, respectively, $\mathrm{KW} ; T_{\mathrm{EM} 1}(s)$ and $n_{\mathrm{EM} 1}(s)$ are the torque and speed of EM1, respectively, and the units are $\mathrm{Nm}$ and $\mathrm{rpm} ; T_{\mathrm{EM} 2}(s)$ and $n_{\mathrm{EM} 2}(s)$ represent the torque and speed of EM2, respectively, and the units are Nm and rpm.

According to the Equations (2)-(4), the output torques of EM1 and EM2 are calculated by:

$$
\begin{aligned}
& T_{\mathrm{EM} 1}(s)=\frac{k(s)}{1+k(s)} \frac{P_{\mathrm{des}}(s)}{n_{\mathrm{EM} 1}(s)} \\
& \mathrm{T}_{\mathrm{EM} 2}(s)=\frac{1}{1+k(s)} \frac{P_{\mathrm{des}}(s)}{n_{\mathrm{EM} 2}(s)}
\end{aligned}
$$

\section{Constraints}

The constraints imposed on the output torque of EM1 and EM2 are less than or equal to the maximum available value. The working status of the dual motors should be the same. It is not allowed that one motor outputs drive force and another feedback energy. Simultaneously, the power of dual motors is limited to extend the battery life. In detail, the constraints can be described as follows.

$$
\begin{gathered}
T_{\mathrm{EM} 1 \mathrm{cmin}}\left(n_{\mathrm{EM} 1}(s)\right) \leq T_{\mathrm{EM} 1}(s) \leq T_{\mathrm{EM} 1 \mathrm{dcmax}}\left(n_{\mathrm{EM} 1}(s)\right) \\
T_{\mathrm{EM} 2 \mathrm{cmin}}\left(n_{\mathrm{EM} 2}(s)\right) \leq T_{\mathrm{EM} 2}(s) \leq T_{\mathrm{EM} 2 \mathrm{dcmax}}\left(n_{\mathrm{EM} 2}(s)\right) \\
\left|P_{\mathrm{EM} 1}(s)\right|+\left|P_{\mathrm{EM} 2}(s)\right| \leq\left|P_{\text {batt_lim }}(s)\right|
\end{gathered}
$$

where $T_{\mathrm{EM} 1 \mathrm{cmin}}$ and $T_{\mathrm{EM} 2 \mathrm{cmin}}$ denote the minimum available torque of EM1 and EM2 under charging, respectively, and the unit is $\mathrm{Nm}$; $T_{\mathrm{EM} 1 \mathrm{dcmax}}$ and $T_{\mathrm{EM} 2 \mathrm{dcmax}}$ are the maximum available torque of EM1 and EM2 under discharge, respectively, and the unit is Nm; $P_{\text {batt_lim }}(s)$ denotes the critical power of battery charging or discharging, KW.

3. Objective Function

The torque distribution control strategy aims to minimize the power, so the total power is treated as the objective function. The formula is as follows:

$$
\begin{aligned}
& P_{\mathrm{out}}(s)=\frac{k(s)}{1+k(s)} \frac{P_{\mathrm{des}}(s)}{\eta_{\mathrm{EM} 1}^{-\operatorname{sigh}\left(P_{\mathrm{des}}(s)\right)}(s)}+\frac{1}{1+k(s)} \frac{P_{\mathrm{des}}(s)}{\eta_{\mathrm{EM} 2}^{-s i n n\left(P_{\operatorname{des}}(s)\right)}(s)} \\
= & \left(\frac{k(s)}{1+k(s)} \frac{1}{\eta_{\mathrm{EM} 1}^{-s i g h\left(P_{\mathrm{des}}(s)\right)}(s)}+\frac{1}{1+k(s)} \frac{1}{\eta_{\mathrm{EM} 2}^{-s i g n\left(P_{\operatorname{des}}(s)\right)}(s)}\right) P_{\mathrm{des}}(s)
\end{aligned}
$$

where $\eta_{\mathrm{EM} 1}(s)$ and $\eta_{\mathrm{EM} 2}(s)$ denote the efficiencies of EM1 and EM2 operating point, respectively; $\operatorname{sign}\left(P_{\mathrm{des}}(s)\right)$ is a symbolic function of $P_{\mathrm{des}}(s)$.

Based on the problem description, it can be found that optimizing dual-motor torque distribution is a non-linear mathematical optimization problem of the scale factor.

\subsubsection{Algorithm Realization}

To obtain the minimum value of the objective function, APSO algorithm is employed to optimize the torque distribution of the dual motors. The APSO is an improved algorithm for PSO, which can avoid the operation consequence falling into the regional optimum. This optimization algorithm can solve multi-dimensional optimization issues and has high computational efficiency and a little amount of mathematics. It can be employed for online real-time optimization in the engineering field. The benefits of the APSO algorithm in enhancing energy utilization and reducing usage costs have been adequately confirmed $[22,28-30]$. The procedure of algorithm completion is portrayed in Figure 4 . It 
consists of five phases. The detailed calculation process of the algorithm and the preference of parameter values can be found in [22].

\section{Adaptive initialization}

Taking the highest efficiency operating point of the motors as the starting point, the particle position is initialized with equal proportional distribution to avoid falling into the local optimum in each operating mode.

\section{Update inertia weight and learning factors}

Nonlinear reduction inertia weights and dynamic learning factors update.

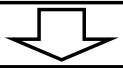

\section{Particle swarm fitness calculation}

Use the objective function as the solution algorithm to calculate the fitness of each particle.

$$
\begin{aligned}
& P_{\text {out }}(s)=\frac{k(s)}{1+k(s)} \frac{P_{\text {des }}(s)}{\eta_{\mathrm{EM} 1}^{-s i n}\left(P_{\mathrm{des}}(s)\right)}+\frac{1}{1+k(s)} \frac{P_{\mathrm{des}}(s)}{\eta_{\mathrm{EM} 2}^{-s i n\left(P_{\mathrm{des}}(s)\right)}(s)} \\
& =\left(\frac{k(s)}{1+k(s)} \frac{1}{\eta_{\mathrm{EM} 1}^{-s i g n\left(P_{\mathrm{des}}(s)\right)}(s)}+\frac{1}{1+k(s)} \frac{1}{\eta_{\mathrm{EM} 2}^{-s i g n\left(P_{\mathrm{des}}(s)\right)}(s)}\right) P_{\mathrm{des}}(s)
\end{aligned}
$$

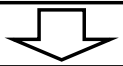

\section{Update pbest, gbest, and particle position velocity}

Use iterative equations to update speed, position, and determine the value of pbest, gbest at each stage.

$$
\begin{array}{cc}
\text { Velocity update } & v_{\mathrm{id}}^{k-1}=\omega v_{\mathrm{id}}^{k-1}+c_{1} r_{1}\left(\text { pbest }_{\mathrm{id}}-x_{\mathrm{id}}^{k-1}\right)+c_{2} r_{2}\left(\text { gbest }_{\mathrm{d}}-x_{\mathrm{id}}^{k-1}\right) \\
\text { Position update } & x_{\mathrm{id}}^{k}=x_{\mathrm{id}}^{k-1}+v_{\mathrm{id}}^{k-1}
\end{array}
$$

\section{Determine the output result}

Determine whether the number of iterations or optimization results meet the preset conditions.

Figure 4. Optimization process of APSO algorithm.

\subsection{Torque Arbitration}

The primary purpose of the arbitration module is to finally determine the torque of two motors based on the status of the dual motor, the flag of the mode switching action, the battery status, the vehicle status, and other related variables. Condition monitoring and fault diagnosis functions are also included in the arbitration module, which plays a critical part in the driving stability of vehicles.

\section{Results and Discussion}

In order to verify the performance of eDMTP and energy management strategy, the mining vehicle equipped with eDMTP and typical real operation environment are selected as the input conditions for verification (Figure 5). Table 1 lists the main operating parameters. 


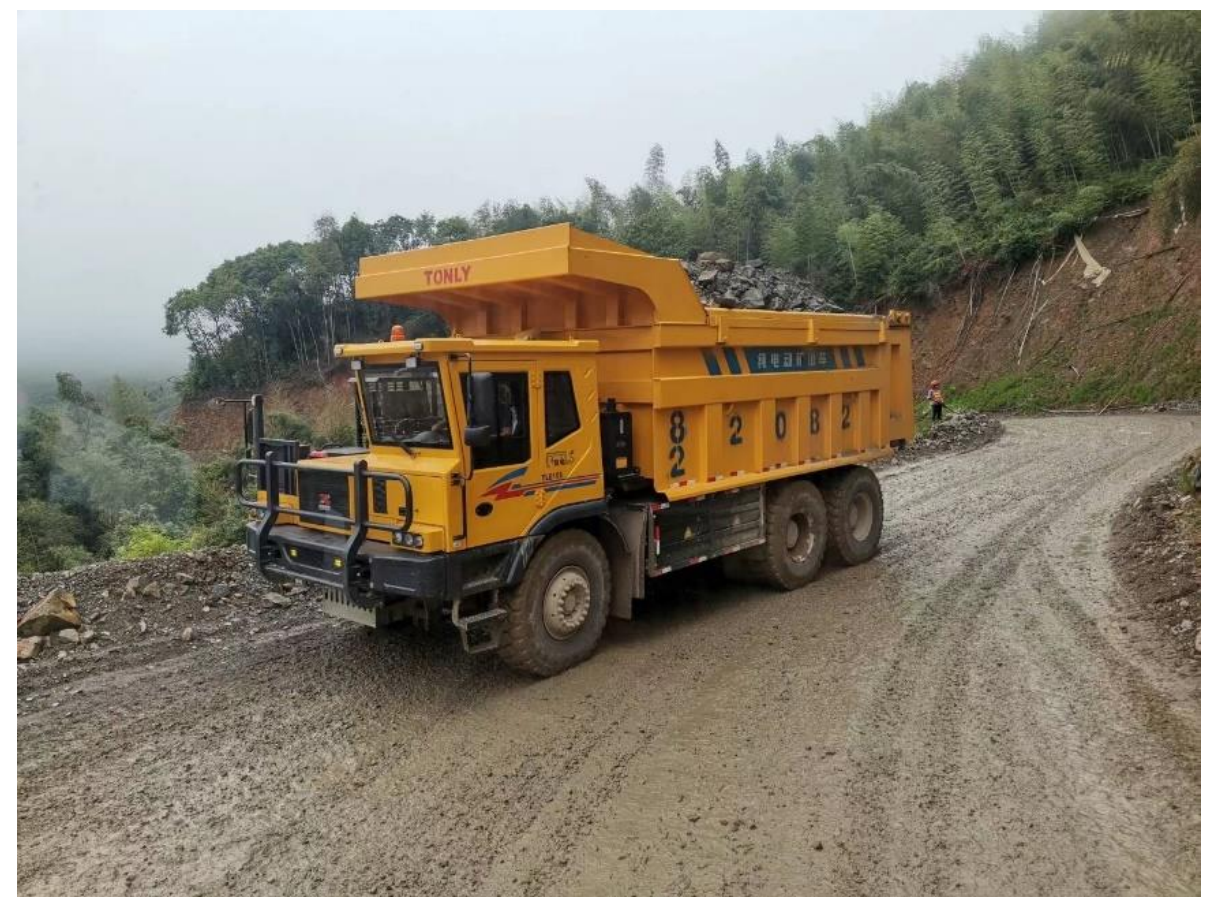

Figure 5. Mining vehicle equipped with eDMTP.

Table 1. Main operating parameters.

\begin{tabular}{cc}
\hline Parameter & Value \\
\hline Cross mass $(\mathrm{kg})$ & 35,000 \\
Cross mass $(\mathrm{kg})$ & 90,000 \\
Dynamic rolling radius $(\mathrm{m})$ & 0.667 \\
Frontal area $\left(\mathrm{m}^{2}\right)$ & 13.6 \\
Air drag coefficient & 0.68 \\
Rolling resistance coefficient & 0.2 \\
Inertia coefficient & 1.1 \\
Gravitational acceleration $\left(\mathrm{m} / \mathrm{s}^{2}\right)$ & 9.8 \\
Final drive ratio & 14.1 \\
\hline
\end{tabular}

Figure 6 displays the actual speed curve. Figure 7 shows the torque request of eDMTP, which is calculated from the vehicle's required torque and the corresponding actual operating mode is shown in Figure 8. It can be found from Figure 8 that there are five operating modes (M2-M6) in addition to neutral mode involved in the whole process. The running time in escape mode M2 is about $40 \mathrm{~s}$, indicating that the vehicle has a greater torque demand during this period. M4 and M5 account for a relatively large amount of working time among the four normal modes, demonstrating that these two modes are more efficient under the premise of satisfying dynamics. Due to the limitation of working environment, the actual speed of the vehicle is relatively low and M7 is not used during operation. 


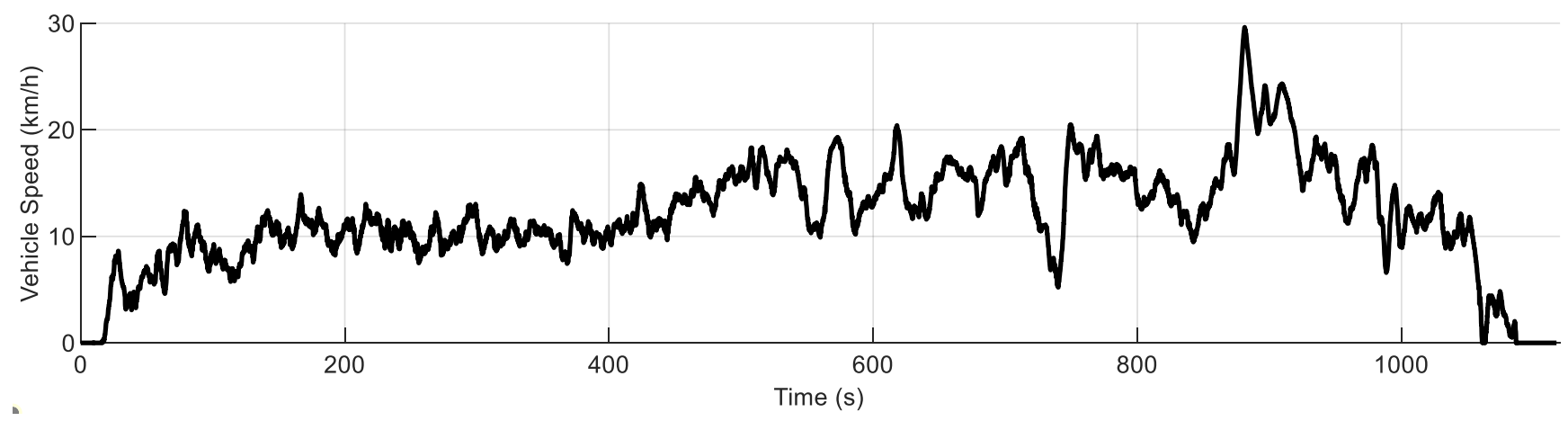

Figure 6. Vehicle speed-time curve.

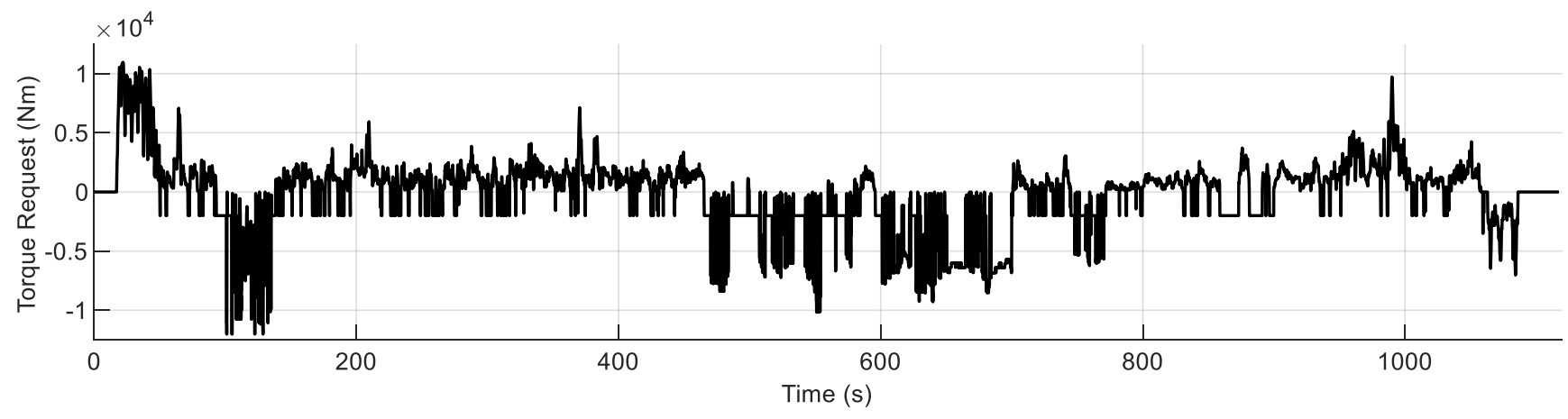

Figure 7. Torque request-time curve.

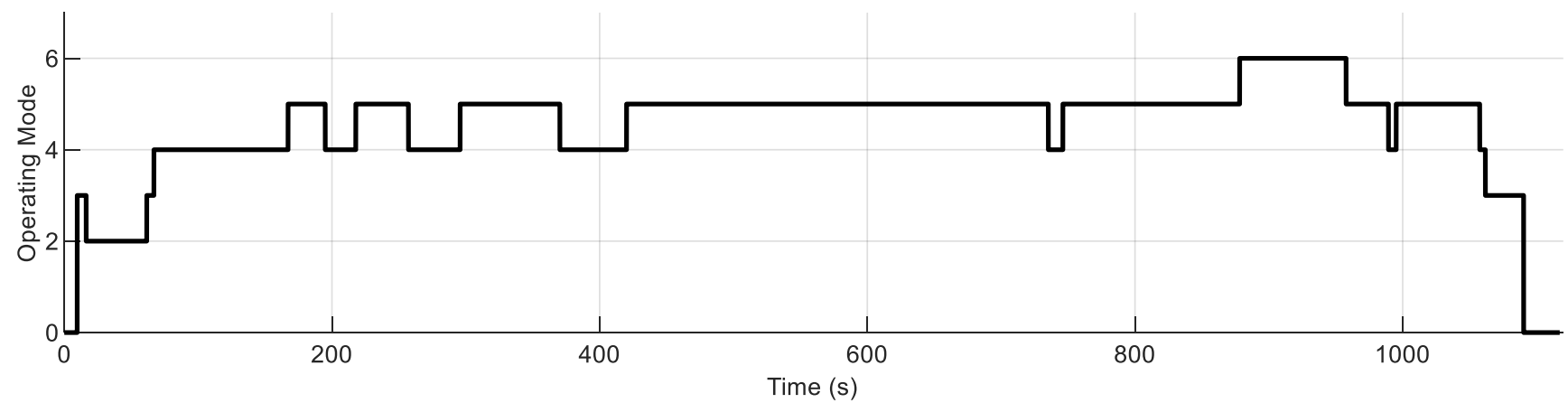

Figure 8. Operating mode-time curve of eDMPT.

The operating points of EM1 and EM2 in the M3-M6 are shown in Figures 9-12. It can be detected from Figure 9 that M3 is the starting mode under the normal condition, and its operating points are mainly concentrated in the low speed and low torque area. This is unavoidable because the overall vehicle efficiency is usually lower in the starting phase under normal driving conditions. As can be seen from Figures 10-12, the operating points of the two motors in M4-M6 are both in and around the high-efficiency area, which illustrates the effectiveness of the energy management strategy in improving transmission efficiency. 


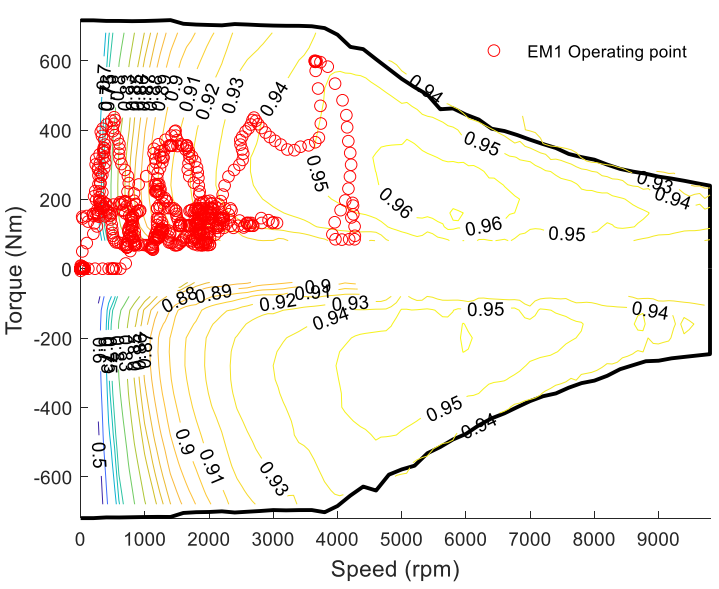

(a)

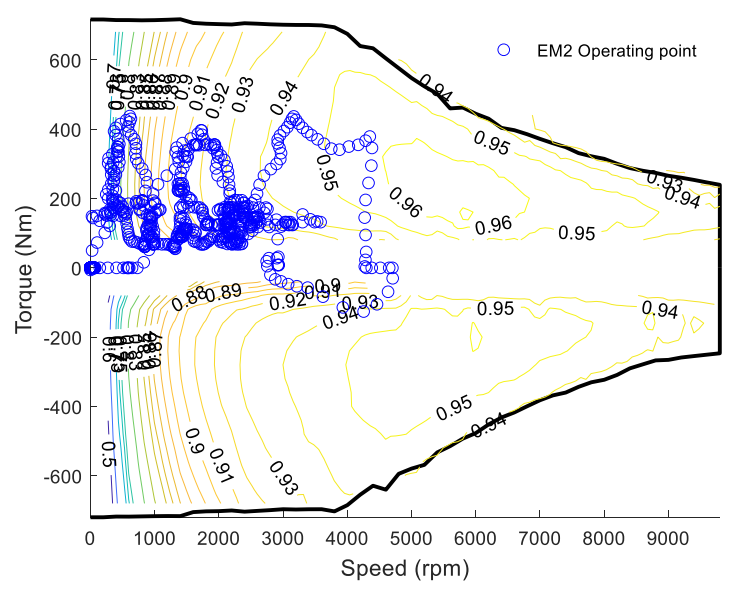

(b)

Figure 9. EM1 (a) and EM2 (b) operating points in M3.

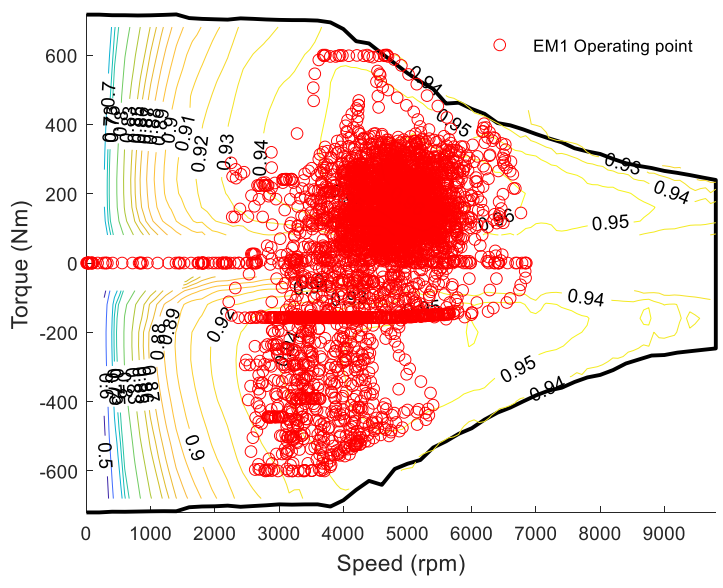

(a)

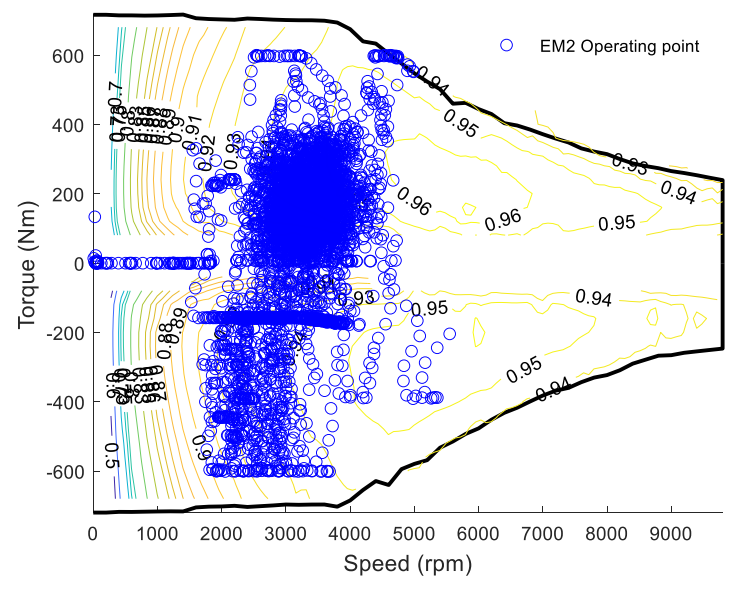

(b)

Figure 10. EM1 (a) and EM2 (b) operating points in M4.

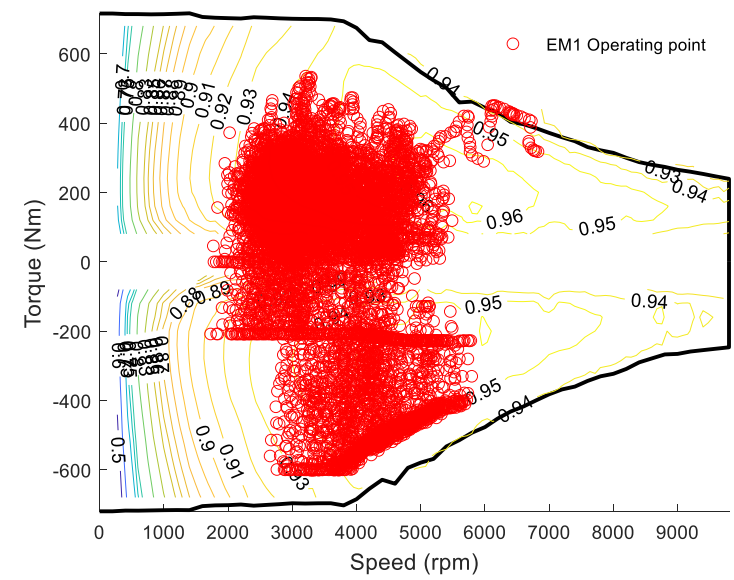

(a)

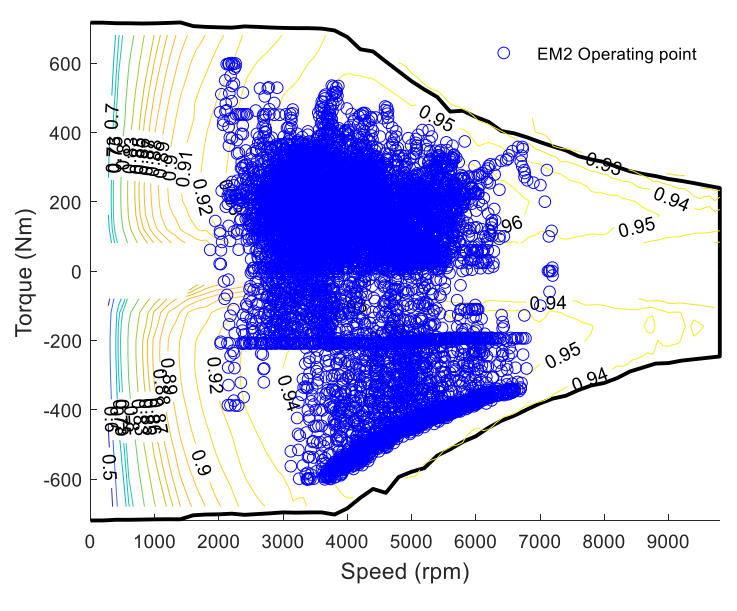

(b)

Figure 11. EM1 (a) and EM2 (b) operating points in M5. 


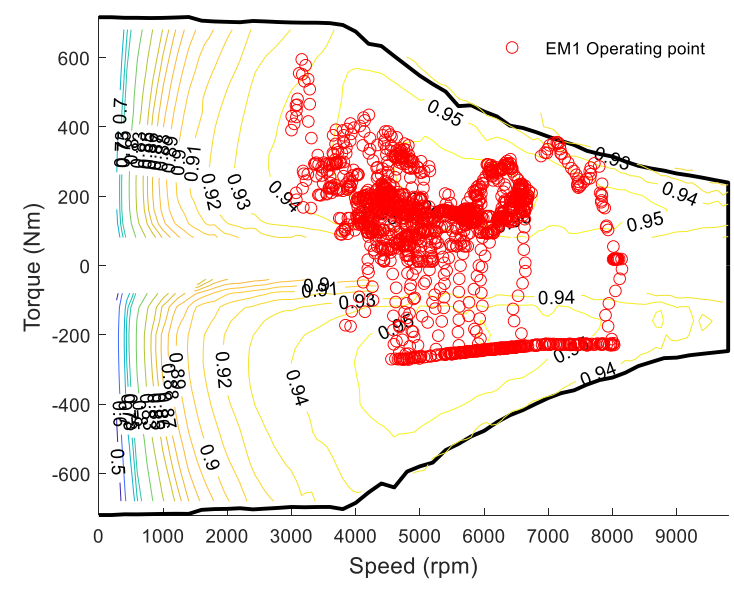

(a)

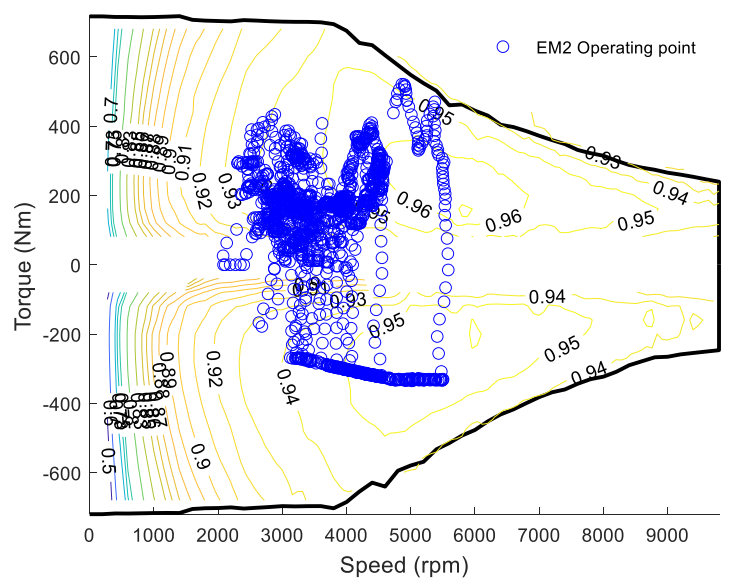

(b)

Figure 12. EM1 (a) and EM2 (b) operating points in M6.

\section{Conclusions}

This study proposes a new dual-motor transmission for heavy commercial vehicles. The structural principle, operating mode, and mode switching logic are analyzed. The eDMTP transmission not only solves the problem of power interruption but also realizes the decoupling of the operating points of the two drive motors, which provides the possibility to improve system efficiency. In addition, the software architecture of the energy management strategy is designed, and a real-time torque split control strategy is developed based on APSO. The proposed control strategy and the performance of eDMTP are verified by actual vehicle tests. In the future, the multi-objective online algorithm will be considered to optimize the operating mode and the torque of the dual motor at the same time so as to further improve the energy saving of the vehicle.

Author Contributions: Conceptualization, J.L., X.X., S.W. and P.D.; methodology, J.L., W.G. and P.D.; formal analysis, S.W., T.F. and W.G.; investigation, T.F. and X.X.; resources, X.X. and S.W.; data curation, J.L. and P.D.; writing — original draft preparation, J.L.; writing-review and editing, J.L. and P.D.; visualization, W.G.; supervision, X.X. and S.W.; project administration, X.X. and S.W.; funding acquisition, X.X. and S.W. All authors have read and agreed to the published version of the manuscript.

Funding: This research was funded by the 2025 Science and Technology Innovation Program of Ningbo “R\&D of Key Technologies for Electric Vehicle Range Extenders"(Grant No. 2019B10111), Key Science and Technology Innovation Project of Shandong Province (Grant No.: 2020CXGC011004, 2019JZZY010913), 2025 Science and Technology Innovation Program of Ningbo (Grant No.: 2020Z026, 2020Z027, 2020Z028), Key Science and Technology Project of Guangxi Province (Grant No.: AA19254013), and Primary Research and Development Plan of Zhejiang Province (Grant No.: 2021C01140).

Institutional Review Board Statement: Not applicable.

Informed Consent Statement: Not applicable.

Data Availability Statement: Data available on request due to privacy restrictions.

Acknowledgments: Thank you to all reviewers for their suggestions to make this paper better.

Conflicts of Interest: The authors declare no conflict of interest.

\section{References}

1. Zacharof, N.; Fontaras, G.; Ciuffo, B.; Tansini, A.; Prado-Rujas, I. An estimation of heavy-duty vehicle fleet $\mathrm{CO}_{2}$ emissions based on sampled data. Transp. Res. Part D Trans. Environ. 2021, 94, 102784. [CrossRef]

2. Smallbone, A.; Jia, B.; Atkins, P.; Roskilly, A. The impact of disruptive powertrain technologies on energy consumption and carbon dioxide emissions from heavy-duty vehicles. Energy Convers. Manag. X 2020, 6, 100030. [CrossRef] 
3. Zhang, X.; Zhu, X. Autonomous path tracking control of intelligent electric vehicles based on lane detection and optimal preview method. Expert Syst. Appl. 2019, 121, 38-48. [CrossRef]

4. Xu, X.; Dong, P.; Liu, Y.; Zhang, H. Progress in automotive transmission technology. Automot. Innov. 2018, 1, 187-210. [CrossRef]

5. Tian, Y.; Zhang, N.; Zhou, S.; Walker, P. Model and gear shifting control of a novel two-speed transmission for battery electric vehicles. Mech. Mach. Theory 2020, 152, 103902. [CrossRef]

6. Huang, J.; Zhang, J.; Huang, W.; Yin, C. Optimal speed synchronization control with disturbance compensation for an integrated motor-transmission powertrain system. J. Dyn. Syst. Meas. Control 2019, 141, 041001. [CrossRef]

7. Wang, X.; Li, L.; He, K.; Liu, Y.; Liu, C. Position and force switching control for gear engagement of automated manual transmission gear-shift process. J. Dyn. Syst. Meas. Control 2018, 140, 81010-81019. [CrossRef]

8. Xu, X.; Liang, J.; Hao, Q.; Dong, P.; Wang, S.; Guo, W.; Liu, Y.; Lu, Z.; Geng, J.; Yan, B. A Novel Electric Dual Motor Transmission for Heavy Commercial Vehicles. Automot. Innov. 2021, 4, 34-43. [CrossRef]

9. Nguyen, C.T.; Walker, P.D.; Zhang, N. Shifting strategy and energy management of a two-motor drive powertrain for extendedrange electric buses. Mech. Mach. Theory 2020, 153, 103966. [CrossRef]

10. Lin, X.; Zhang, G.; Wei, S. Velocity prediction using Markov Chain combined with driving pattern recognition and applied to Dual-Motor Electric Vehicle energy consumption evaluation. Appl. Soft Comput. 2021, 101, 106998. [CrossRef]

11. Zhang, L.; Li, L.; Qi, B.; Song, J. High speed stability electromechanical coupling control for dual-motor distributed drive electric vehicle. J. Mech. Eng. 2015, 51, 12. [CrossRef]

12. Zhang, L.; Yang, L.; Guo, X.; Yuan, X. Stage-by-phase multivariable combination control for centralized and distributed drive modes switching of electric vehicles. Mech. Mach. Theory 2020, 147, 103752. [CrossRef]

13. Park, J.Y.; Heo, S.J.; Kang, D.O. Development of torque vectoring control algorithm for front wheel driven dual motor system and evaluation of vehicle dynamics performance. Int. J. Auto. Technol. KOR 2020, 21, 1283-1291. [CrossRef]

14. De Carlo, M.; Mantriota, G. Electric vehicles with two motors combined via planetary gear train. Mech. Mach. Theory 2020, 148, 103789. [CrossRef]

15. Mantriota, G.; Reina, G. Dual-motor planetary transmission to improve efficiency in electric vehicles. Machines 2021, 9, 58. [CrossRef]

16. Wu, J.; Zhang, N. Driving mode shift control for planetary gear based dual motor powertrain in electric vehicles. Mech. Mach. Theory 2021, 158, 104217. [CrossRef]

17. Sarvaiya, S.; Ganesh, S.; Xu, B. Comparative analysis of hybrid vehicle energy management strategies with optimization of fuel economy and battery life. Energy 2021, 228, 120604. [CrossRef]

18. Zhou, Y.; Ravey, A.; Péra, M.-C. Multi-objective energy management for fuel cell electric vehicles using online-learning enhanced Markov speed predictor. Energy Convers. Manag. 2020, 213, 112821. [CrossRef]

19. Shafaati, M.; Alam, M.; Ahmad, F.; Shariff, S.; Asghar, M. Artificial intelligence-based performance optimization of electric vehicle-to-home (V2H) energy management system. Int. J. Sustain. Transp. 2020, 1, 115-125.

20. Sánchez, M.; Delprat, S.; Hofman, T. Energy management of hybrid vehicles with state constraints: A penalty and implicit Hamiltonian minimization approach. Appl. Energy 2020, 260, 114149. [CrossRef]

21. Guo, R.; Sun, Z. Novel research for energy management of plug-in hybrid electric vehicles with dual motors based on pontryagin's minimum principle optimized by reinforcement learning. SAE Int. 2021, 168424. [CrossRef]

22. Zheng, Q.; Tian, S.; Zhang, Q. Optimal torque split strategy of dual-motor electric vehicle using adaptive nonlinear particle swarm optimization. Math. Probl. Eng. 2020, 2020, 1-21. [CrossRef]

23. Guo, C.; Cao, D.; Qiao, Y.; Yang, Z.; Hou, Z. Energy management strategy of extended-range electric bus based on model predictive control. SAE Int. J. Commer. Veh. 2021, 14, 229-238. [CrossRef]

24. Liu, Y.; Liu, J.; Zhang, Y.; Wu, Y.; Ye, M. Rule learning based energy management strategy of fuel cell hybrid vehicles considering multi-objective optimization. Energy 2020, 207, 118212. [CrossRef]

25. Wang, H.; Huang, Y.; Lv, C.; Khajepour, A. A global optimal energy management system for hybrid electric off-road vehicle. $S A E$ Int. J. Commer. Veh. 2017, 10, 524-531. [CrossRef]

26. Wang, X.; He, H.; Sun, F.; Zhang, J. Application study on the dynamic programming algorithm for energy management of plug-in hybrid electric vehicles. Energies 2015, 8, 3225-3244. [CrossRef]

27. Xu, X.; Zhao, J.; Zhao, J.; Shi, K.; Dong, P.; Wang, S.; Liu, Y.; Guo, W.; Liu, X. Comparative study on fuel saving potential of series-parallel hybrid transmission and series hybrid transmission. Energy Convers. Manag. 2022, 252, 114970. [CrossRef]

28. Tan, S.; Yang, J.; Zhao, X.; Yang, W.; Khajepour, A. Power distribution strategy development and optimization of an integrated dual-motor transmission for electric dump truck. IEEE T. Transp. Electr. 2021, 7, 1964-1975. [CrossRef]

29. Chen, S.; Hung, Y.; Wu, C.; Huang, S. Optimal energy management of a hybrid electric powertrain system using improved particle swarm optimization. Appl. Energy 2015, 160, 132-145. [CrossRef]

30. Reza, S.; Ali, R.; Mohammad, E.; Ali, R. Intelligent Energy Management and Multi-Objective Power Distribution Control in Hybrid Micro-grids based on the Advanced Fuzzy-PSO Method. ISA T. 2020, 112, 119-213. 